\title{
Cornea Regeneration as an Alternative to Human Donor Transplantation
}

\author{
Isabelle Brunette, ${ }^{1}$ Emilio I Alarcon ${ }^{2}$ and May Griffith ${ }^{3}$
}

1. Professor and Senior Consultant, Department of Ophthalmology, University of Montreal, Canada; 2. Assistant Professor, University of Ottawa Heart Institute, Canada; 3. Professor in Regenerative Medicine, Department of Clinical and Experimental Medicine, Linköping University, Sweden

DOI: http://doi.org/10.17925/EOR.2015.09.02.111

\begin{abstract}
There is a need for an alternative to human donor corneas as the availability of good-quality tissues remains limited, with this situation potentially worsening as the population in many countries is progressively ageing. There have been numerous attempts to develop corneal equivalent as alternatives to donated human corneas as well as prostheses. In this short review, we focus on the efforts in bioengineering implants that promote regeneration by Canadian researchers, including our current team of authors. The examples of technologies developed that we describe include biomaterials that allow for partial regeneration of corneal tissue, self-assembled cornea constructs and cell-free corneal implants that promoted regeneration when evaluated in clinical trials in Europe.
\end{abstract}

\section{Keywords}

Cornea, biomaterials, prostheses, regeneration, self-assembly, implants, clinical studies

Disclosure: Patents related to the recombinant human collagen (RHCIII) and RHCIII-MPC biomaterials formulation described in this study has been filed and assigned to the Ottawa Hospital Research Institute (OHRI) and are currently licensed to Eyegenix Inc. and LinkoCare Life Science. Isabelle Brunette, Emilio I Alarcon and May Griffith have no affiliations with these companies. No funding was received in the publication of this article.

Open Access: This article is published under the Creative Commons Attribution Noncommercial License, which permits any non-commercial use, distribution, adaptation and reproduction provided the original author(s) and source are given appropriate credit.

Received: 30 October 2015 Accepted: 20 November 2015 Citation: European Ophthalmic Review, 2015;9(2):111-14

Correspondence: May Griffith, Department of Clinical and Experimental Medicine, Cell Biology Building, Floor 10 Linköping University, SE-58598 Linköping, Sweden.

E: May.Griffith@liu.se

The human cornea is the optically clear window of the eye and its main refractive component that focuses light to the retina allowing vision. Thus, the optical transparency of the cornea is critical for optimal vision. Injury or diseases that cause irreversible loss of transparency lead to vision loss and eventually blindness. Globally, it is estimated that 4.9 million individuals have bilateral corneal blindness ${ }^{1}$ while 23 million are estimated to be unilaterally cornea blind. ${ }^{2}$ Cornea transplantation with human donor corneas is the only extensively accepted treatment to restore eyesight. In fact, human cornea is one of the most transplanted tissues worldwide, with more than 53,000 corneal grafts performed in 2013 in North America alone. ${ }^{3}$ However, it is becoming increasingly difficult for Eye Banks worldwide to meet the growing demand for good-quality donated corneas, which is in part due to population ageing. According to the Eye Bank Association, of America, the number of corneal transplantations performed in the US has increased by $50.9 \%$ since 2005 , a number that has increased faster than the number of potential donors., ${ }^{3,4}$ Thus, donor tissue shortage has become a growing concern for most countries. ${ }^{5-8}$

Artificial corneas made from traditional plastics, such as the AlphaCor ${ }^{\circledR}$ Keratoprosthesis are available, but these do not completely biointegrate into the host cornea. The Boston Keratoprostheses and osteo-odontokeratoprosthesis (OOKP) have a plastic optic but a corneal rim or tooth as the interface with the patient's remaining cornea. These two biological interfaced devices have been reported to be among the most successful prostheses, but are still reserved for end-stage disease,, 10 as they restore only minimal function and require lifetime antibiotic therapy and immune suppression. They also increase the risk of other sight-threatening conditions, such as glaucoma, which require an additional surgical procedure, such as the placement of a shunt to alleviate eye pressure.

Currently, when a cornea requires replacement by transplantation, the pathological tissue is surgically removed and replaced by a donated human eye bank cornea that is sutured or glued in place. The overall success rate is high in many countries, such as Sweden, with rejection at only $10-15 \%$ over the first 2 years post-operative. What is not as commonly reported is that transplantation results are still suboptimal. For example, immune rejection is problematic in some cases and can lead to graft failure. Appositioning of the graft tissue could result in astigmatism if the fit is not precise. Sutures are often left on for up to 1 year as graft integration is slow, and these can become loose and cause infection. For high-risk patients with inflammation and severe pathologies (e.g. chemical burns, autoimmune diseases, previously rejected grafts, limbal keratoplasties), complications and failure rates have been as high as $49 \% .{ }^{11-13}$ Multiple surgeries are often needed and patients face the prospect of complete vision loss as each subsequent transplant fails.

\section{Bioengineered Substitutes as Cornea Replacements}

While corneal surgeons have practically reached a plateau for optimising outcomes following traditional corneal transplantation using human donor corneas, biomaterials technology potential offers a range of new opportunities for solving current limitations, as new materials and designs are possible. Other potential advantages that bioengineered corneal substitutes might have over donated human corneas include the following: 


\section{Figure 1: Slit Lamp Biomicroscopy Images of the Corneas of All 10 Patients at 4 years after Grafting with a Biosynthetic Implant}
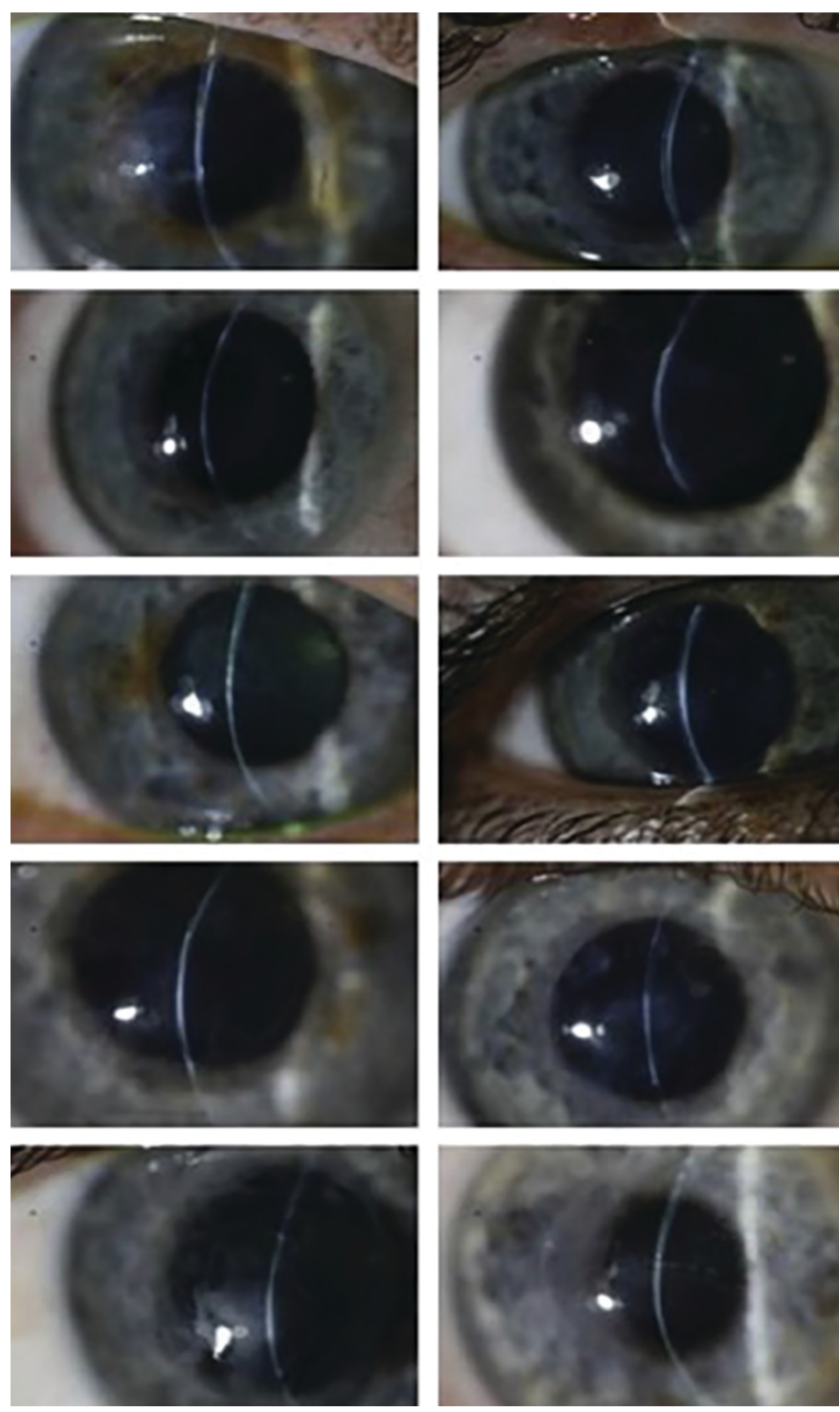

Reproduced with permission from Fagerholm et al., 2014.23

Large-scale production of biosynthetic implants offers a powerful potential solution to the severe shortage of human donor corneas worldwide:

1. Good manufacturing practices (GMPS) eliminate the need for expensive screening for diseases transmitted by donors and for tissue quality assessment;

2. Acellular (cell-free), corneal substitutes circumvent immune rejection induced by allogeneic cells, thus reducing the risk of graft failure and eliminating the need for chronic post-operative immunosuppression;

3. The 3D shape of the corneal substitute can be tailored the same way contact lenses and intraocular lenses are shaped, aiming at more successful post-operative refractive results. Corneal shape is of primary importance, as it determines most of the eye's total refractive power and, hence, visual acuity; ${ }^{14}$

4. The biomechanical properties of the material can be adapted to the clinical need, e.g. they can be made softer, more elastic or stronger and firmer, or they can allow for transportation of fluid and solids and therefore not affect intraocular pressure measurements; and

5. These biomaterials can be designed to specifically interact with cells, micro-organisms or molecules by the release of bioactives.

We and others have reviewed the various keratoprostheses and bioengineered corneal substitutes that have been developed as replacements for pathological corneas and/or to promote regeneration. In this article, we review only the contributions of Canadian researchers to the development of technologies for promoting corneal regeneration as an alternative to donor transplantation. One of these bioengineered corneal implants reached clinical evaluation in Europe, in a clinical trial conducted in Sweden.

\section{Keratoprostheses with Regenerative Capability}

The traditional keratoprostheses as mentioned above have focused on replacement of minimal function to allow vision. Sheardown and co-workers have focused on keratoprostheses that have allowed partial regeneration, by modification of biomaterials with cell adhesion peptides and growth factors. ${ }^{15,16}$ The following are examples of biomaterials developed for use as keratoprostheses.

In Aucoin et al., ${ }^{17}$ poly(dimethyl siloxane) (PDMS) surfaces were modified by covalent attachment of combinations of cell adhesion peptides that were derived from laminin and fibronectin. The peptides studied included the commonly studied YIGSR and its synergistic peptide PDSGR from laminin, and fibronectin-derived RGDS and PHSRN. Statistical analysis of the experimental adhesion results suggested that the concentrations of YIGSR, RGDS and PHSRN used, as well as the synergistic effect of YIGSR and PDSGR, had significant effects on cell attachment and proliferation. The use of surface modification with multiple peptides resulted in superior adhesion and proliferation of corneal epithelial cells over surfaces modified with single peptides only. These results showed that the use of combinations of synergistic peptides potentially results in enhanced cell surface interactions.

In Liu and Sheardown, ${ }^{18}$ the high water permeability of hydrogels, modelled with poly ( $\mathrm{N}$-isopropyl acrylamide), was combined with the high oxygen permeability of poly dimethyl siloxane (silicone) in an interpenetrating network (IPN). The resulting IPN possessed mechanical strength that was superior to that of each individual polymer. Incorporation of interfacial agents resulted in better transparency than that of the individual polymers and, overall, these materials demonstrated adequate corneal epithelial cell compatibility. While promising, there has been limited testing with these materials under in vivo conditions.

\section{Self-assembled Corneal Constructs}

The team of Auger, Germain and their co-workers at the Laboratoire D'Organogénèse Expérimentale (LOEX) have developed a fundamental technique for developing self-assembly of tissues by inducing cultured target cells to synthesise their own extracellular matrix (ECM). For corneal constructs, ascorbic acid was used to stimulate the production of collagen and other ECM molecules by cultured stromal cells. Sheets of secreted ECM can be stacked together to form a thicker stack and an epithelium is seeded on top. The morphology and function of these sheets reproduces many of the main features of the human cornea. ${ }^{19}$ 
Figure 2: Corneal Features in a Healthy, Unoperated Subject, Alongside those of Operated Patients, at 24 Months after Implantation of a Biosynthetic Cornea or a Human Donor Cornea

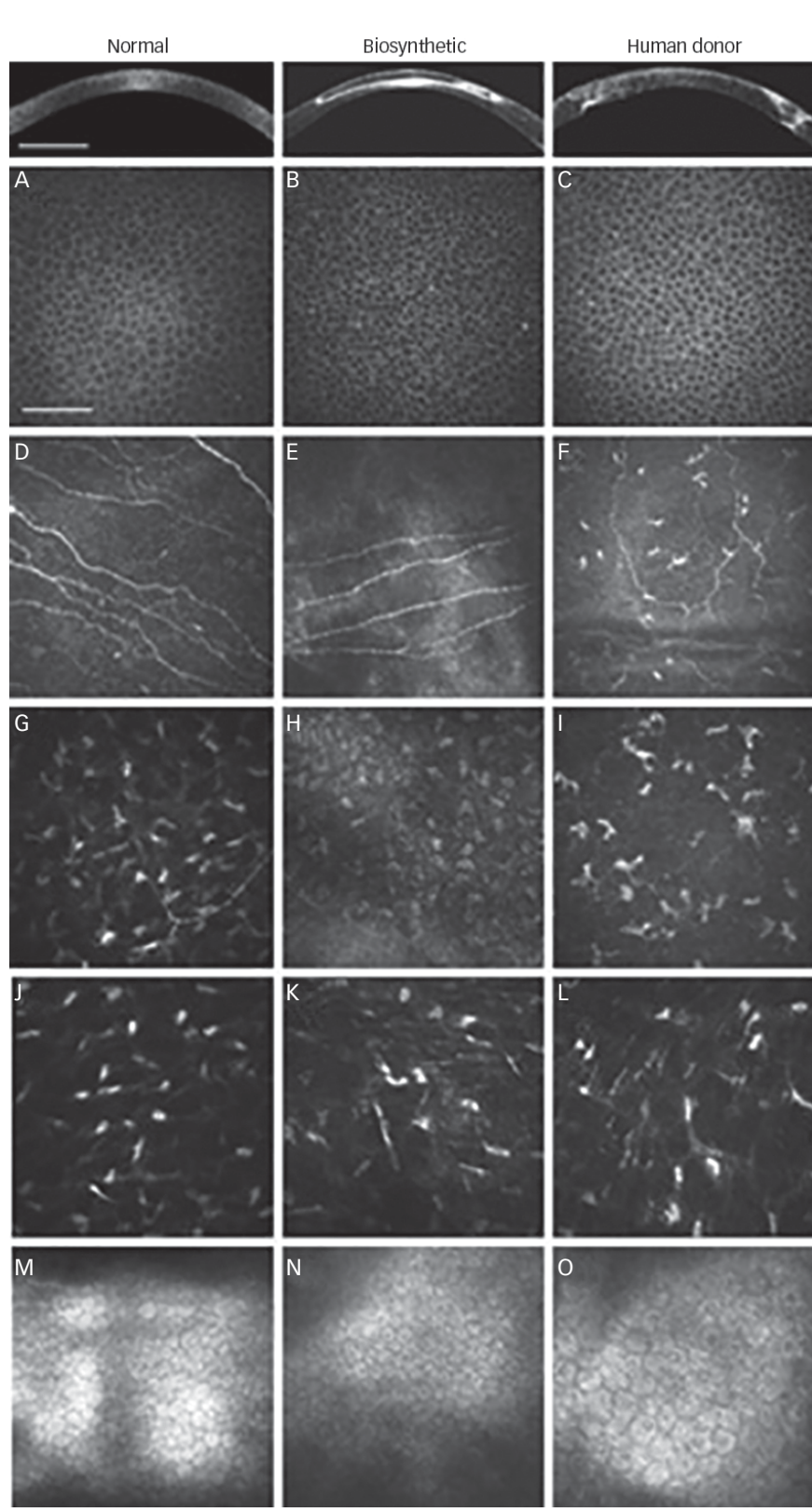

(Top row) Anterior segment optical coherence tomography (ASOCT) images of a healthy cornea, biosynthetic implant and human donor transplant by penetrating keratoplasty $(P K)$. Areas of wound-healing activity exhibit high reflectivity (white areas). (A to O) in vivo confocal microscopy (IVCM) images. Intact epithelium of the unoperated cornea (A), regenerated corneal epithelial cells on the implant surface $(B)$ and regenerated epithelium of the penetrating graft (C). Regenerated nerves $(E)$ at the subbasal epithelium in an implanted cornea were parallel and morphologically similar to the normal cornea (D), whereas regenerated subbasal nerves were also observed in a cornea transplanted with human donor tissue (F). Anterior stromal cell (keratocyte) nuclei (G to I) and posterio keratocytes (J to L) were present, with varying density, in all corneas. The endothelium (M to 0 ) in all corneas exhibited a characteristic mosaic pattern. Scale bars, $2 \mathrm{~mm}$ (ASOCT), $100 \mathrm{~mm}$ (IVCM). Reproduced with permission from Fagerholm et al., 2010.22

To date, self-assembled cornea constructs have been developed that reproduce one or more layers of the human cornea. ${ }^{20,21} \mathrm{~A}$ tissueengineered stroma replacement was tested in cats recently and at 4 months post-operation, all grafts were stable and clear. ${ }^{21}$ These implants were re-innervated, showing comparable touch sensitivity to pre-operation levels. Histological evaluation showed a lamellar structure similar to that of a healthy cornea.

\section{Figure 3: Corneas of All Three Patients Before and After Implantation with Tectonic Grafts of RHCIII-MPC}

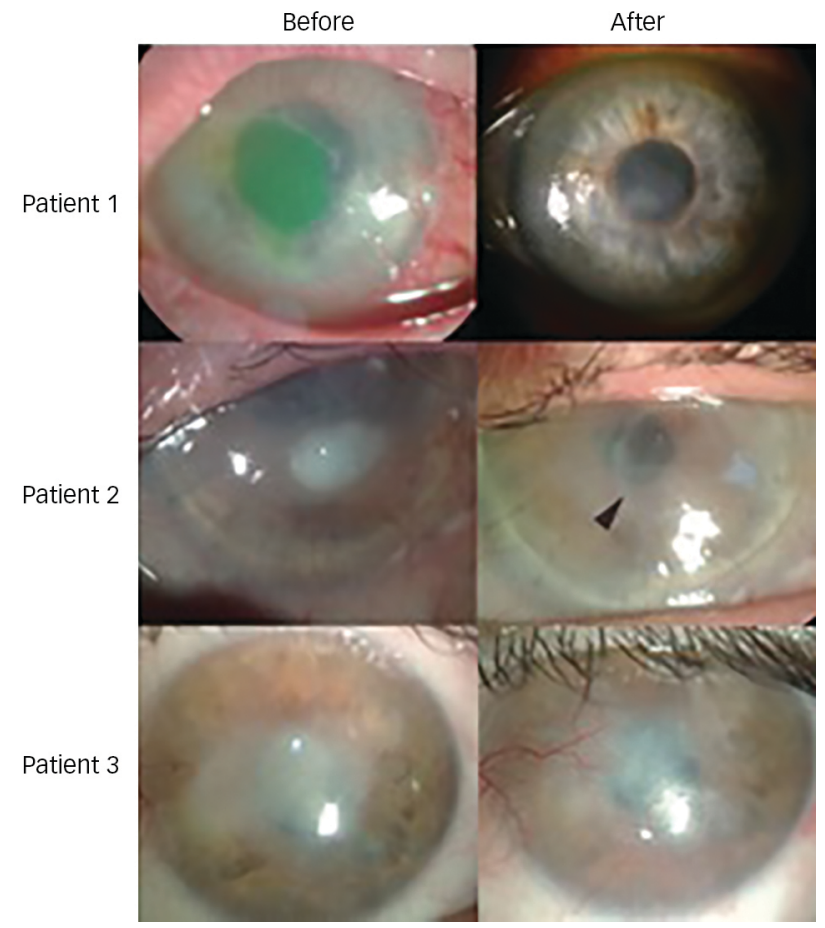

Patient 1's cornea had an ulcer overlying a vascularised stroma. The green fluorescein staining delineates the large area of eroded epithelium. At 12 months post-operation, the cornea is intact and relatively clear. Patient 2 had a failed $8.5 \mathrm{~mm}$ diameter graft with a persistent ulcer and dense stromal opacification in the visual zone prior to surgery. A small $4 \mathrm{~mm}$ implant was grafted into the ulcerated area of the failed graft (arrowhead) and has remained relatively clear after 12 months. Patient 3 had an opacity with an unstable epithelial surface and vascularised stroma prior to surgery. At 9 months postoperation, while the implant remained clear, the ingrowing conjunctiva has left the surface hazy. Reproduced with permission from Buznyk et al., 2015.25

\section{First Demonstration of Regeneration of the Human Cornea Promoted by a Cell-free Implant}

Members of our team recently published the 4-year follow-up results of a phase I clinical study where transparent biosynthetic analogues of human corneal stromal extracellular matrices, comprising carbodiimide-crosslinked recombinant human collagen type III (RHCIII), were implanted in the first 10 human patients. ${ }^{22}$ We showed that the implants were stably integrated without immunosuppression. Unlike donor corneas, they did not attract antigen-presenting dendritic cells (see Figure 1). Furthermore, by mimicking the corneal ECM, they promoted in-growth of the patients' own corneal epithelial cells, nerves and stromal keratocytes to regenerate a neo-cornea (see Figure 2). ${ }^{22,23}$ This was a major step in corneal transplantation as we demonstrated for the first time that the human cornea is capable of regeneration, and that biomaterials can be used to stimulate the regeneration as an alternative to donor human allografts, giving hope to patients who were not transplanted due to a severe worldwide shortage of donor tissues.

Further development of these implants included the use of riboflavin and UVA light to potentially crosslink or weld the implants into the corneas of hosts. In a study conducted by Wand et al. ${ }^{24}$ RHCIII corneal implants were successfully crosslinked into excised porcine and rabbit eyes. While further optimisation is required to address shrinkage that was noted after crosslinking, nevertheless, in the future, this technique could help reduce suture-related complications, 


\section{Cataract and Cornea}

such as haze formation and surface irregularity after grafting of bioengineered collagen-based implants.

\section{Bioengineered Cornea Implants for High-risk Patients}

The first RHCIII substitutes were implanted in non-inflamed corneas. In order to ensure that the next generation of implants will resist the insults of inflamed eyes, including collagenases, dryness and neovascularisation, which typically lead to ulceration, melting and opacification of the graft, the RHCIII implants were reinforced. A second network of 2-methacryloyloxyethyl phosphorylcholine (MPC) crosslinked with polyethylene (glycol) diacrylate (PEGDA) was added to form an interpenetrating network. The first RHCIII-MPC implants were developed in Canada, but optimisation for clinical evaluation was conducted in sweden. The optimised generation of RHCIII-
MPC implants were tested in a pilot clinical study conducted in the Filatov Institute for Eye Diseases and Tissue Therapy, Odessa, Ukraine. Three patients with persistent ulceration and erosions of the cornea surface and who were at high risk of rejection of human donor corneas were implanted with RHCIII-MPC as tectonic grafts. All three patients showed relief of the symptoms of pain, irritation and photophobia. The implants were still stably integrated at 9 and 12 months in the patients (see Figure 3). Two of three patients also showed improvement in vision. ${ }^{25}$

\section{Conclusion}

The field of cornea regeneration is one that is active and many groups are working on the development of new technologies. We have shown how several technologies developed by Canadian researchers have now been enhanced by ongoing European research including clinical evaluation.
1. Oliva MS, Schottman T, Gulati M, Turning the tide of corneal blindness, Indian J Ophthalmol, 2012;60:423-7.

2. Lohiya S, Attal R, Bokariya P, Eye banking and corneal transplantation in tertiary care hospital located in rural area IOSR J Pharmacy, 2014;4:11-6.

3. Eye Bank Association of America, 2013 Eye Banking Statistical Report. 2014: Washington, DC

4. Eye Bank Association of America, 2009 Eye Banking Statistical Report. 2010: Washington, DC

5. Mack RJ, Mason P, Mathers WD, Obstacles to donor eye procurement and their solutions at the University of lowa, procurement and their solt

6. Poinard C, Tuppin P, Loty B, Delbosc B, The French national waiting list for keratoplasty created in 1999: patient registration, indications, characteristics, and turnover J Fr Ophthalmol, 2003:26:911-9.

7. Rasouli M, Caraiscos VB, Slomovic AR, Efficacy of routine notification and request on reducing corneal transplantation wait times in Canada, Can J Ophthalmol, 2009;44:31-5.

8. Reinhard T, Bohringer D, Bogen A, Sundmacher R, The transplantation law: a chance to overcome the shortage of corneal grafts in Germany?, Transplant Proc, 2002;34:1322-4.

9. Avadhanam VS, Smith HE, Liu C, Keratoprostheses for corneal blindness: a review of contemporary devices, Clin Ophthalmol, 2015;9:697-720.

10. Avadhanam VS, Liu CS, A brief review of Boston type-1 and osteo-odonto keratoprostheses, Br J Ophthalmol,

\section{5;99:878-87}

11. Guilbert E, Bullet J, Sandali O, et al., Long-term rejection incidence and reversibility after penetrating and lamell keratoplasty, Am J Ophthalmol, 2013;155:560-9 e2.

12. Weisbrod DJ, Sit M, Naor J, Slomovic AR, Outcomes of repeat penetrating keratoplasty and risk factors for graft failure, Cornea, 2003;22:429-34.

13. Yu AL, Kaiser M, Schaumberger M, et al., Donor-related risk factors and preoperative recipient-related risk factors for graft failure, Cornea, 2014:33:1149-56.

14. Gatinel D, Topographie cornéenne, 2011, Paris. Available at: http://www.gatinel.com/chirurgie-refractive/bilanat: http://Www.gatinel.com/chirurgie-refractive/bilan-
preoperatoire//'etude-de-la-cornee-topographie-corneennemesure-de-l'epaisseur-et-de-la-resistance-biomecaniquemesure-de-l' epaisseur-et-de-la-resistance-bi
corneenne/ (accessed 28 October 2015).

15. Klenkler BJ, Dwivedi D, West-Mays JA, Sheardown H, Corneal epithelial cell adhesion and growth on EGF-modified aminated PDMS, J Biomed Mater Res A, 2010;93:1043-9.

6. Princz MA, Sheardown $\mathrm{H}$, Heparin-modified dendrimer cross-linked collagen matrices for the delivery of basic fibroblast growth factor (FGF-2), J Biomater sci Polym Ed, 2008;19:1201-18

17. Aucoin L, Griffith CM, et al., Interactions of corneal epithelial cells and surfaces modified with cell adhesion peptide combinations, J Biomater Sci Polym Ed, 2002;13:447-62.

18. Liu L, Sheardown H, Glucose permeable poly (dimethyl siloxane) poly ( $\mathrm{N}$-isopropyl acrylamide) interpenetrating networks as ophthalmic biomaterials, Biomaterials, 2005;26:233-44

19. Proul $x$ S, d'Arc Uwamaliya J, Carrier P, et al., Reconstruction of a human cornea by the self-assembly approach of tissue engineering using the three native cell types, Mol Vis, 2010;16:2192-201.

20. ProulX S, Brunette I, Methods being developed for preparation, delivery and transplantation of a tissueengineered corneal endothelium, Exp Eye Res, 2012;95:68-75.

21. Boulze Pankert M, Goyer B, Zaguia F, et al., Biocompatibility and functionality of a tissue-engineered living corneal stroma and functionality of a tissue-engineered living corneal str 2014;55:6908-20.

22. Fagerholm P, Lagali NS, Merrett K, et al., A biosynthetic alternative to human donor tissue for inducing corneal regeneration: 24-month follow-up of a phase 1 clinical study, Sci Transl Med, 2010;2:46ra6

23. Fagerholm P, Lagali NS, Ong JA, et al., Stable cornea regeneration four years after implantation of a cell-free recombinant human collagen scaffold, Biomaterials, 2014;35:2420-7.

24. Wand K, Neuhann R, Ullmann A, et al., Riboflavin-UV--a crosslinking for fixation of biosynthetic corneal collagen implants, Cornea, 2015;34:544-9.

25. Buznyk O, Pasyechnikova N, Islam MM, et al., Bioengineered corneas grafted as alternatives to human donor corneas in three high-risk patients, Clin Transl Sci, 2015;8:558-62. 